\title{
BMJ Open Efficacy of different surgical approaches on survival outcomes in patients with early-stage cervical cancer: protocol for a multicentre longitudinal study in China
}

Xiaopei Chao, Ming Wu, Shuiqing Ma, Xianjie Tan, Sen Zhong, Xiaochen Song, Lei Li (D)

To cite: Chao X, Wu M, Ma S, et al. Efficacy of different surgical approaches on survival outcomes in patients with earlystage cervical cancer: protocol for a multicentre longitudinal study in China. BMJ Open 2020;10:e038020. doi:10.1136/ bmjopen-2020-038020

- Prepublication history and additional material for this paper are available online. To view these files, please visit the journal online (http://dx.doi org/10.1136/bmjopen-2020038020).

Received 25 February 2020 Revised 29 May 2020 Accepted 20 July 2020
Check for updates

(C) Author(s) (or their employer(s)) 2020. Re-use permitted under CC BY-NC. No commercial re-use. See rights and permissions. Published by BMJ.

Obstetrics and Gynecology, Peking Union Medical College Hospital, Beijing, China

Correspondence to Dr Lei Li; lileigh@163.com

\section{ABSTRACT}

Introduction Recent studies have revealed that the oncological survival outcomes of minimally invasive radical hysterectomy (MIRH) are inferior to those of abdominal radical hysterectomy (ARH) in early-stage cervical cancer, but the potential reasons are unclear.

Methods and analysis Each expert from 28 study centres participating in a previously reported randomised controlled trial (NCT03739944) will provide successive eligible records of at least 100 patients who accepted radical hysterectomy for early-stage cervical cancer between 1 January 2009 and 31 December 2015. Inclusion criteria consist of a definite pathological evaluation of stages IA1 (with positive lymphovascular space invasion), IA2 and IB1 according to the International Federation of Gynecology and Obstetrics 2009 staging system and a histological subtype of squamous cell carcinoma, adenocarcinoma or adenosquamous carcinoma. The primary endpoint is 5-year disease-free survival between the MIRH and ARH groups. The secondary endpoints include the MIRH learning curves of participating surgeons, 5-year overall survival between the MIRH and ARH groups, survival outcomes according to surgical chronology, surgical outcomes and sites of recurrence and potential risk factors that affect survival outcomes. A subgroup analysis in patients with tumour diameter less than $2 \mathrm{~cm}$ will follow the similar flow diagram.

Ethics and dissemination This study has been approved by the Institutional Review Board of Peking Union Medical College Hospital (registration no. JS-1711), and is also filed on record by all other 27 centres. The results will be disseminated through community events and peerreviewed journals.

Trial registration number NCT03738969

\section{INTRODUCTION}

Cervical cancer is the fourth most common malignancy in women worldwide. ${ }^{1}$ In addition, $85 \%$ of patients with cervical cancer are from developing countries, and cervical cancer is the second leading cause of cancer-related death in women. ${ }^{1}$ In China, according to a conservative estimate, the total incidence and mortality of cervical cancer in 2015 were 98900

\section{Strengths and limitations of this study}

- With the advantage of the enormous number of patients with cervical cancer receiving radical hysterectomy in China and the diversity of operation methods in involved centres, as well as the systematic follow-up system, this study can be implemented as soon as possible, and the results will reflect the general essence of such findings.

- This study will be of great importance to the comprehensive consideration and practice of surgeons, and the learning curve of each surgeon for treatment of cervical cancer will also be taken into consideration of selecting appropriate surgical routes.

- The main limitation is the retrospective nature of the study based on the data in medical records. Disease-specific and treatment-specific issues affecting quality of life cannot be adequately assessed. The emphasis on the individual surgeon's experience and skill probably limits generalisation to all surgeons.

and 30500 cases, respectively, ${ }^{2}$ accounting for $28 \%$ of the total number of new cases of cervical cancer worldwide. ${ }^{3}$ Radical hysterectomy (RH) with pelvic lymphadenectomy is the standard surgical treatment for patients with early-stage cervical cancer. ${ }^{4}$ Previously published guidelines ${ }^{45}$ indicate that either laparotomy (open surgery) or minimally invasive surgery (MIS) is an acceptable approach to treat RH in patients with early-stage (IA2 to IIA) cervical cancer. In numerous retrospective studies, although MIS and abdominal RH (ARH) have comparable 5-year diseasefree survival (DFS) and overall survival (OS) rates ${ }^{6-13}$ MIS also has better shorter-term quality of life (QoL) and improved surgical outcomes, ${ }^{11} 12$ 14-23 even in elderly patients. ${ }^{24}$ Nevertheless, one prospective randomised study by Ramirez et $a l^{13}$ and one retrospective epidemiological study by Melamed et $a t^{25}$ put forward doubts and documented that ARH for 
early-stage cervical cancer is superior to minimally invasive RH (MIRH) regarding DFS and OS. These findings were also confirmed in a recent population-based survey in England. ${ }^{26}$ It seems that MIS may have a reputation for this inferior trend, which is significantly different from the situation observed in early-stage uterine, colorectal or gastric cancer. ${ }^{27-30}$ These studies caused great controversy over the surgical approaches of cervical cancer worldwide, but they certainly changed the idea of the best approach to offer early-stage cervical cancer patients undergoing surgical treatment. ${ }^{251-34}$ However, the causes of the inferior survival outcomes in the MIS group are still unknown. Many possible reasons for the inferiority of MIS have been debated, such as different radicality, incision methods, ethnic differences, tumour size as a selection criterion, learning curves, data completeness, circulating $\mathrm{CO}_{2}$ levels and the usage of manipulators. ${ }^{35}$ Hence, we performed a randomised controlled trial (RCT) in 28 Chinese centres (RACC, NCT03739944) to evaluate the survival outcomes of patients who underwent MIRH and ARH and to explore potential risk factors. ${ }^{36}$ This trial is based on a participating expert-centred design rather than a study site-centred design. ${ }^{36}$

However, the two most important questions that arose from the RACC study (NCT03739944) await answers. One is the safety of MIRH for patients with early-stage cervical cancer that was inferior, reported in the Laparoscopic Approach to Cervical Cancer (LACC) trial and other studies. ${ }^{132526}$ The other is how to qualify the experiences of participating surgeons. In one retrospective study on RH procedures performed by one of the participating surgeons, we found that the learning curve probably explains the disadvantage of MIRH, as the survival outcomes of patients who underwent the first dozens of MIRH procedures performed by the surgeon were inferior to those of patients who underwent ARH. ${ }^{37}$ The current study proposes a multicentre longitudinal study in China to answer these two questions, since the study centre and surgeons who participated in NCT03739944 are the same as those who will participate in the current study.

The quality of the participating surgeons can be determined by the MIRH learning curve. Few studies have considered the influencing factors involving surgeons and their learning curves, ${ }^{37}{ }^{38}$ and research defining learning curves in gynaecological oncology surgery is limited. Surgeons can be proficient after 20 cases of robotic-assisted RH (RRH) and continually improve between 50 and 70 cases. ${ }^{39-41}$ Mastery of laparoscopic RH (LRH) requires experience in at least 25 and up to 50 cases. ${ }^{42-44}$ According to Hwang et $a l^{45}$ the learning period for LRH and lymph node dissection to reach a turning point was calculated to be 40 cases. The systematic review found a slow learning curve required for a surgeon to gain expertise in laparoscopically assisted vaginal $\mathrm{RH}^{46}$ These studies support the necessity of evaluating the learning curves in a demanding surgical modality.
In addition, two retrospective reports of a large series showed that ARH and MIRH had different survival outcomes in patients with a tumour diameter $>2 \mathrm{~cm}$, while patients with a tumour diameter $<2 \mathrm{~cm}$ experienced superior outcomes to those with a tumour diameter $>2 \mathrm{~cm} .{ }^{25}$ Kim $e t a l^{47}$ also reported that MIRH was not a poor prognostic factor for patients with stage IB1 cervical cancer and cervical masses $\leq 2 \mathrm{~cm}$ in size. Some authors also argued that the reduction in survival in patients receiving MIRH could be due to tumour manipulation and subsequent cancer spread. ${ }^{48}$

In general, based on the participating surgeons of an $\mathrm{RCT}^{36}{ }^{6}$ we will perform this multicentre longitudinal study to compare the survival outcomes of patients undergoing ARH and MIRH and to qualify the learning curves of participating surgeons. Other essential issues, including tumour diameters and definite surgical procedures which were considered as potential risk factors, will be also evaluated in this study.

\section{Aims and objectives}

Primary objectives

1. The primary aim of this longitudinal study is to analyse the 5-year DFS rates of patients with early-stage cervical cancer receiving different surgical routes, including MIS (RRH or LRH) versus ARH, in all enroled patients and in patients with tumour diameter $<2 \mathrm{~cm}$.

\section{Secondary objectives}

1. The learning curves of participating surgeons will be presented as their qualification proof.

2. The 5-year OS rates between patients in the MIRH and ARH groups.

3. The 5-year DFS and OS rates according to chronology in general and individually.

4. To compare the time of surgery, estimated blood loss during surgery, volume of transfusion, length of hospital stay, perioperative complications and postoperative complications between the MIRH and ARH groups.

5. To describe patterns of recurrence, including sites, tumour burden and biomarkers.

6. To investigate relevant factors guiding a surgeon's choice about the best surgical approach for patients who may still benefit from MIS without worsening their survival outcomes. These factors include pathological characteristics, energy devices, uterine manipulators, adjuvant treatment, follow-up protocols, nerve-sparing procedures and expert characteristics.

These analysis will be performed in all enroled patients and in patients with tumour diameter $<2 \mathrm{~cm}$.

\section{METHODS AND ANALYSIS \\ Study design}

This is a retrospective, multicentre study comparing the efficacy of different surgical approaches on the surgical and oncological survival outcomes in Chinese patients with early-stage cervical cancer receiving RH. A total of 


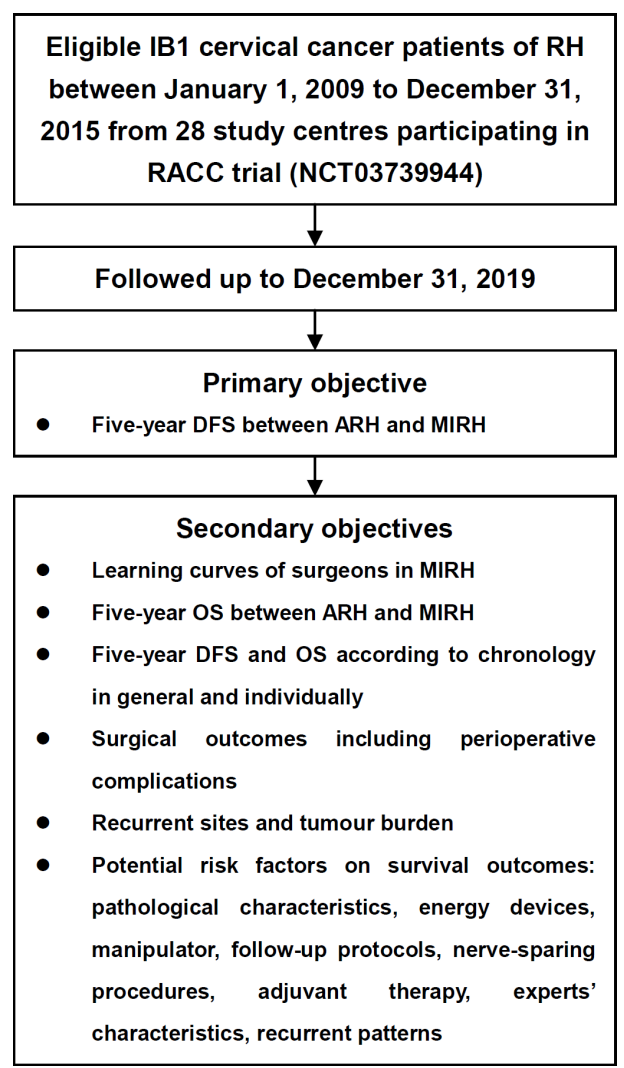

Figure 1 Flow diagram of the study. $\mathrm{ARH}$, abdominal $\mathrm{RH}$; DFS, disease-free survival. MIRH, minimally invasive $\mathrm{RH}$; OS, overall survival. $\mathrm{RH}$, radical hysterectomy.

28 Chinese centres (online supplementary file 1), same as the RACC trial ${ }^{36}$ will collect patients' medical records. Each expert included in this study will provide the medical records of at least 100 consecutive patients who were hospitalised between 1 January 2009 and 31 December 2015 , according to a predefined template. These patients were followed up until 31 December 2019, to guarantee a follow-up of at least 4 years. The study flow chart is illustrated in figure 1.

\section{Recruitment and eligibility}

Patients' medical records will be collected based on the following eligibility criteria.

\section{Inclusion criteria}

1. Patients with stage IA1 (with lymphovascular space invasion (LVSI)), IA2 or IB1 cervical cancer according to the International Federation of Gynecology and Obstetrics 2009 staging system ${ }^{49}$ and an Eastern Cooperative Oncology Group performance status ${ }^{50}$ of 0 to 1 .

2. Histological subtypes of squamous cell carcinoma, adenocarcinoma, and adenosquamous carcinoma.

3. Patients older than 18 years at diagnosis.

4. Patients had complete epidemiological and clinicopathological data recorded.

5. All enroled patients underwent surgery performed by the experts designated in the research centre.
Exclusion criteria

1. Patients with a histological subtype of neuroendocrine carcinoma, clear cell carcinoma, serous cell type carcinoma or metastatic carcinoma.

2. Patients with stage IA1 disease without LVSI or clinically advanced disease (stages IB2-IV).

3. Pregnant women.

4. Patients receiving neoadjuvant chemotherapy or radiotherapy before $\mathrm{RH}$.

5. Patients with a history of pelvic radiotherapy, pelvic reconstruction, and brain/spinal cord diseases.

6. Patients with a positive HIV status, autoimmune disorders and systemic disease (such as hormone treatment diseases, severe liver and kidney dysfunction) or a severe mental illness and pre-existing cancer diagnosis.

7. Patients without integrated medical records, such as details on epidemiology, surgery and pathology.

\section{Interventions}

Study centre and surgeon selection

The selection and determination of study centres and surgeons were described previously. ${ }^{36}$ All the surgeons (ie, the principal investigators) from all study centres listed in online supplementary file 1 approved the study protocol and signed a research agreement form.

\section{Surgical treatment}

The surgical procedures performed have been described previously ${ }^{36}$ : these consisted of $\mathrm{RH}$, bilateral salpingooophorectomy and pelvic lymphadenectomy (the resection of sentinel lymph nodes also needed clear notification), with/without concomitant para-aortic lymph node dissection. For young patients requiring the preservation of ovaries, bilateral salpingectomy was performed along with ovary suspension to the peritoneum above the level of the anterior superior spine. All the major procedures, including lymphadenectomy and parametrial resection with or without a nerve-sparing procedure, were primarily performed by one surgeon according to the Piver-Rutledge-Smith classification ${ }^{51}$ or to class B or $\mathrm{C}$ of the Q-M classification. ${ }^{52}$ Patients who converted to laparotomy due to intraoperative complications were allocated to the ARH group. During the review of medical records, the following surgical issues will receive special attention and recording: energy devices, artificial pneumoperitoneum, manipulator usage, vaginal incision methods used during the surgeries and nerve-sparing procedures.

\section{Postsurgical adjuvant treatment}

Postoperative adjuvant therapies, including systematic chemotherapy, radiotherapy and chemoradiotherapy, will be recorded. Therapy regimens, dosages, durations and adverse events will also be addressed.

\section{Sample size calculation}

The primary objective of this study is to explore whether there are differences between MIRH and ARH with respect to DFS. We assume that the 5 year DFS rate will 
be approximately $85 \%$, and the non-inferiority threshold of $5 \%$ is clinically acceptable. The corresponding HR is set at 1.50 based on the significance level, and the power is set at 0.8 . A total of 1140 patients are needed for the MIRH and ARH groups. Considering the possible 20\% loss to follow-up rate, 2850 patients are needed to accomplish the study goal.

In this study, since each participating hospital will be asked to provide the successive eligible records of at least 100 patients, approximately 3000 or more patients could be examined in total to achieve the estimated sample size. However, such estimation does not guarantee a sufficient sample size in the MIRH or ARH group.

\section{Measurement}

The patients' detailed epidemiology, surgical details, pathological characteristics, perioperative complications and follow-up data, as well as postoperative adjuvant therapy data, will be collected by medical staff. All data obtained from domestic research centres will be input into the database by trained medical staff. Details are as follows:

1. Surgical outcomes include estimated blood loss, transfusion, surgical duration and hospital stay after RH.

2. Pathological outcomes include the measurement of critical parameters from available records, consisting of the width or length of the resected parametrium, vagina and uterosacral ligaments under their natural conditions; numbers and locations of harvested lymph nodes; and feasibility of sentinel lymph node biopsy. Due to the results and concerns from LACC trial ${ }^{13}$ and other studies, ${ }^{4753}$ patients with tumour diameter $<2 \mathrm{~cm}$ will be included a subgroup analysis as to guarantee the safety of patients in future trials.

3. Complications will be recorded as intraoperative, perioperative, early $(<4$ weeks $)$ postoperative and delayed postoperative ( 4 weeks to 6 months after $\mathrm{RH}$ ) according to the protocol of the LACC trial, ${ }^{54}$ and severity will be judged by the Common Terminology Criteria for Adverse Events (CTCAE) V.4.03 ${ }^{55}$ Definite complications include intraoperative complications (bladder injury, ureteral injury, bowel injury, vascular injury and obturator nerve injury), postoperative complications (urinary retention with catheterisation, cerebrovascular, pulmonary and renal diseases, ileus, abdominal wound and vault complications, septicemia, thromboembolic complications, pelvic haematoma, lymphocyst formation and/or secondary infection, postoperative vesicovaginal fistula, ureterovaginal fistula, rectovaginal fistula, chylous leakage and lymphedema).

4. Survival outcomes: the time to recurrence will be calculated from the date of surgery to the time the patient is diagnosed with disease recurrence. DFS will be calculated from diagnosis to the date of death from cervical cancer-associated complications and/or cancer progression. OS will be calculated from the date of surgery to the date of death (or the last follow-up date if the patient is alive).

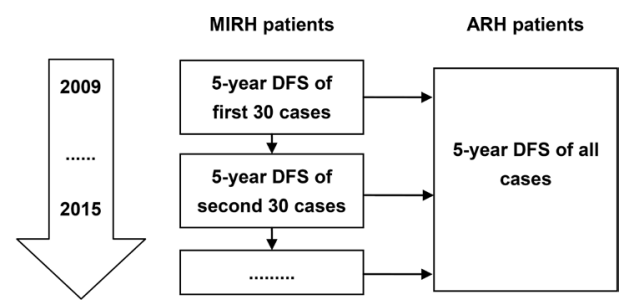

Figure 2 Judgement of learning curves of the study. Patients with MIRH will be divided into subgroups of first 30 cases, second 30 cases and and others. The 5-year DFS of these subgroup of patients with MIRH will be compared with the DFS of patients with ARH to explore the potential differences. The 5-year DFS of subgroup of patients with $\mathrm{MIRH}$ will also be compared with the DFS of first 30 patients with $\mathrm{MIRH}$ to find out the potential plateau of DFS. ARH, abdominal radical hysterectomy. DFS, disease-free survival. $\mathrm{MIRH}$, minimally invasive radical hysterectomy.

5. Participating surgeons will provide personal data including birth date, sex and qualification interval. Their involvement and roles in the RH procedures were confirmed by written documents from their Department of Medical Records. Regarding MIRH, we will clarify the past surgical volumes before the year 2009.

6. Learning curves for MIRH in participating surgeons will be defined as the definite case numbers of MIRH, after which the 5-year DFS rate of patients could achieve a similar level to that of patients with ARH without statistical significance. In our study, we will divide the whole MIRH cohort into the first and the next 30 patents and compare their DFS rates with the DFS rates of the ARH cohort (figure 2). The sequence of patients with will be reported by the Department of Medical Records of the corresponding study centre with written confirmation.

\section{Statistical analysis}

Continuous variables conforming to normal distribution will be described as the means and SDs and compared with parametric methods. Non-parametric tests will be used to assess the categorical data, and discrete variables that do not conform to normal distribution will be summarised as the medians, ranges and IQRs. Survival curves will be generated by the Kaplan-Meier method, and Cox proportional hazards models will be used to estimate the HRs and 95\% CIs for the effect of surgical routes on DFS, progression-free survival and OS. A multivariable analysis of DFS will be performed after adjusting for important pathological risk factors. Statistical analysis will be performed using SPSS V.22.0 (SPSS Inc, Chicago, Illinois, USA). Significance will be set at a p value of 0.05 .

\section{Safety and adverse events}

This trial will be conducted in compliance with this study protocol. Although it is a retrospective study, the Data Safety Monitoring Committee (online supplementary file 2) in the RACC trial ${ }^{36}$ will still receive study data regularly, including complications and survival outcomes. The Committee will review the final data of individual 
surgeon's learning curve, as to make recommendation about further investigation. The concern about the tumour diameter (less or $>2 \mathrm{~cm}$ ) will receive specific attention.

\section{Limitations}

The retrospective nature of the current study is the most important limitation. Disease-specific and treatmentspecific issues affecting QoL cannot be adequately assessed. The emphasis on the individual surgeon's experience and skill probably limits generalisation to all surgeons. How to eliminate or decrease the restriction of the learning curve for young surgeons is critical in oncology education.

\section{Patient and public involvement}

Epidemiological data and clinicopathological characteristics were retrospectively collected by trained medical staff from a third party by reviewing the medical records. Complications related to RH within 6 months were collected as adverse events according to the CTCAE V.4.03.8. ${ }^{55}$ Data on recurrence and mortality were obtained from the medical records or follow-up data by the certificated third party. Sites of recurrence were verified by surgeries and/or imaging evaluations. A detailed follow-up by telephone was also provided to patients with unknown or uncertain survival outcomes. In the current trial, no patients were involved in the design of the study or in the selection of outcome measures.

\section{Ethics and dissemination}

The study will be practiced in compliance with this study protocol. The patient's consent to accept the treatment and the approval of the trial by each study centre will be reviewed and collected by the principle investigator (LL and MW). All procedures performed in the study involving human participants were in accordance with the ethical standards of the institutional and/or national research committee and with the 1964 Declaration of Helsinki and its later amendments or comparable ethical standards.

This is the first version of protocol (Identifier 1.0, 16 February 2019). The protocol will be public on the website of the principle study centre (http://www.pumch.cn). Any important protocol modifications must be communicated to all study centres and investigators, and must be reported to and be approved by the Institutional Review Board of all study centres.

Personal information about potential and enroled participants will be masked by systemic sequence number so as to be collected, shared and maintained in order to protect confidentiality before, during and after the trial. The final data will be available on the online dataset (http://pi.kangruihealth.com) for data sharing after anonymizsation processing.

\section{CONCLUSION}

Due to the complex and complicated characteristics of $\mathrm{RH}$, the learning time needed to achieve comparable survival outcomes is probably long and is equally essential for laparotomy and laparoscopy. Data from large, welldesigned observational studies are urgently needed. After adjusting the bias of surgeons, this study may provide essential evidence of whether MIRH has inferior oncological outcomes to ARH and the potential reasons involved despite of several assumptions, especially in patients with tumour diameter less or $>2 \mathrm{~cm}$. Other important surgical approaches associated with controversial issues, including definite surgical procedures, will also be discussed in this study.

Contributors LL conceived of the original idea for the study, carried out the statistical analysis, edited the paper and was overall guarantor. XC obtained ethical approval and contributed to drafts of the paper. MW, SZ, SM, XT and XS contributed to the study design and commented on drafts of the paper.

Funding This work was supported by the Chinese Academy of Medical Sciences Initiative for Innovative Medicine (CAMS-2017-I2M-1-002). The funders had no role in the study design, data collection and analysis, decision to publish or preparation of the manuscript.

Competing interests None declared.

Patient and public involvement Patients and/or the public were not involved in the design, or conduct, or reporting or dissemination plans of this research.

Patient consent for publication Obtained.

Provenance and peer review Not commissioned; externally peer reviewed.

Open access This is an open access article distributed in accordance with the Creative Commons Attribution Non Commercial (CC BY-NC 4.0) license, which permits others to distribute, remix, adapt, build upon this work non-commercially, and license their derivative works on different terms, provided the original work is properly cited, appropriate credit is given, any changes made indicated, and the use is non-commercial. See: http://creativecommons.org/licenses/by-nc/4.0/.

ORCID iD

Lei Li http://orcid.org/0000-0001-8723-3461

\section{REFERENCES}

1 Bray F, Ferlay J, Soerjomataram I, et al. Global cancer statistics 2018: GLOBOCAN estimates of incidence and mortality worldwide for 36 cancers in 185 countries. CA Cancer J Clin 2018;68:394-424.

2 Chen W, Zheng R, Baade PD, et al. Cancer statistics in China, 2015. CA Cancer J Clin 2016;66:115-32.

3 Chinese guidelines for diagnosis and treatment of cervical cancer 2018 (English version). Chin J Cancer Res 2019;31:295-305.

4 NCCN Clinical Practice Guidelines in Oncology (NCCN Guidelines $®$ ). Cervical cancer version 1, 2020. Available: https://www.nccn.org/ professionals/physician_gls/pdf/cervical.pdf

5 Marth C, Landoni F, Mahner S, et al. Cervical cancer: ESMO clinical practice guidelines for diagnosis, treatment and follow-up. Ann Oncol 2017;28:iv72-83.

6 Lee E-J, Kang H, Kim D-H. A comparative study of laparoscopic radical hysterectomy with radical abdominal hysterectomy for earlystage cervical cancer: a long-term follow-up study. Eur J Obstet Gynecol Reprod Biol 2011;156:83-6.

7 Malzoni M, Tinelli R, Cosentino F, et al. Total laparoscopic radical hysterectomy versus abdominal radical hysterectomy with lymphadenectomy in patients with early cervical cancer: our experience. Ann Surg Oncol 2009;16:1316-23.

8 Nam J-H, Park J-Y, Kim D-Y, et al. Laparoscopic versus open radical hysterectomy in early-stage cervical cancer: long-term survival outcomes in a matched cohort study. Ann Oncol 2012;23:903-11.

9 Sert BM, Boggess JF, Ahmad S, et al. Robot-Assisted versus open radical hysterectomy: a multi-institutional experience for early-stage cervical cancer. Eur J Surg Oncol 2016;42:513-22. 
10 Shah CA, Beck T, Liao JB, et al. Surgical and oncologic outcomes after robotic radical hysterectomy as compared to open radical hysterectomy in the treatment of early cervical cancer. J Gynecol Oncol 2017;28:e82.

11 Wang Y-zhou, Deng L, Xu H-cheng, et al. Laparoscopy versus laparotomy for the management of early stage cervical cancer. BMC Cancer 2015;15:928.

12 Cao T, Feng Y, Huang Q, et al. Prognostic and safety roles in laparoscopic versus abdominal radical hysterectomy in cervical cancer: a meta-analysis. J Laparoendosc Adv Surg Tech A 2015;25:990-8.

13 Ramirez PT, Frumovitz M, Pareja R, et al. Minimally invasive versus abdominal radical hysterectomy for cervical cancer. N Engl J Med 2018;379:1895-904.

14 Guo J, Yang L, Cai J, et al. Laparoscopic procedure compared with open radical hysterectomy with pelvic lymphadenectomy in early cervical cancer: a retrospective study. Onco Targets Ther 2018:11:5903-8.

15 Jin Y-M, Liu S-S, Chen J, et al. Robotic radical hysterectomy is superior to laparoscopic radical hysterectomy and open radical hysterectomy in the treatment of cervical cancer. PLoS One 2018;13:e0193033.

16 Lawrie TA, Liu H, Lu D, et al. Robot-Assisted surgery in gynaecology. Cochrane Database Syst Rev 2019;4:CD011422.

17 Frumovitz M, dos Reis R, Sun CC, et al. Comparison of total laparoscopic and abdominal radical hysterectomy for patients with early-stage cervical cancer. Obstet Gynecol 2007;110:96-102.

18 Shazly SAM, Murad MH, Dowdy SC, et al. Robotic radical hysterectomy in early stage cervical cancer: a systematic review and meta-analysis. Gynecol Oncol 2015;138:457-71.

19 Ditto A, Martinelli F, Bogani G, et al. Implementation of laparoscopic approach for type $B$ radical hysterectomy: a comparison with open surgical operations. Eur J Surg Oncol 2015;41:34-9.

20 Mendivil AA, Rettenmaier MA, Abaid LN, et al. Survival rate comparisons amongst cervical cancer patients treated with an open, robotic-assisted or laparoscopic radical hysterectomy: a five year experience. Surg Oncol 2016;25:66-71.

21 Zeng YC, Ching SSY, Loke AY. Quality of life measurement in women with cervical cancer: implications for Chinese cervical cancer survivors. Health Qual Life Outcomes 2010;8:30.

22 Gallotta V, Conte C, Federico A, et al. Robotic versus laparoscopic radical hysterectomy in early cervical cancer: a case matched control study. Eur J Surg Oncol 2018;44:754-9.

23 Corrado G, Vizza E, Legge F, et al. Comparison of different surgical approaches for stage IB1 cervical cancer patients: a Multiinstitution study and a review of the literature. Int $J$ Gynecol Cancer 2018;28:1020-8.

24 Gallotta V, Conte C, D'Indinosante M, et al. Robotic surgery in elderly and very elderly gynecologic cancer patients. J Minim Invasive Gynecol 2018:25:872-7.

25 Melamed A, Margul DJ, Chen L, et al. Survival after minimally invasive radical hysterectomy for early-stage cervical cancer. $N$ Engl J Med 2018;379:1905-14.

26 Athanasios F, Afrodite N, Effstratios P, et al. Co-Expression of bone morphogenetic protein 6 with estrogen receptor a in endometriosis. Arch Gynecol Obstet 2012;285:1001-7.

27 Fleshman J, Sargent DJ, Green E, et al. Laparoscopic colectomy for cancer is not inferior to open surgery based on 5-year data from the cost Study Group trial. Ann Surg 2007;246:655-64.

28 Bonjer HJ, Deijen CL, Abis GA, et al. A randomized trial of laparoscopic versus open surgery for rectal cancer. N Engl J Med 2015;372:1324-32

29 Walker JL, Piedmonte MR, Spirtos NM, et al. Recurrence and survival after random assignment to laparoscopy versus laparotomy for comprehensive surgical staging of uterine cancer: gynecologic Oncology Group LAP2 study. J Clin Oncol 2012;30:695-700.

30 Janda M, Gebski V, Davies LC, et al. Effect of total laparoscopic hysterectomy vs total abdominal hysterectomy on diseasefree survival among women with stage I endometrial cancer: a randomized clinical trial. JAMA 2017;317:1224-33.

31 Kimmig R, Ind T. Minimally invasive surgery for cervical cancer: consequences for treatment after LACC study. J Gynecol Oncol 2018;29:e75.

32 Naumann RW. Minimally invasive radical hysterectomy has many benefits compared with open radical hysterectomy: will the LACC trial cause the premature demise of this procedure? J Minim Invasive Gynecol 2019;26:379-80.
33 Leitao MM. The LACC trial: has minimally invasive surgery for earlystage cervical cancer been dealt a knockout punch? Int J Gynecol Cancer 2018;28:1248-50.

34 Park JY, Nam JH. How should gynecologic oncologists react to the unexpected results of LACC trial? J Gynecol Oncol 2018;29:e74.

35 Köhler C, Schneider A, Marnitz S, et al. The basic principles of oncologic surgery during minimally invasive radical hysterectomy. $J$ Gynecol Oncol 2020;31:e33.

36 Chao X, Li L, Wu M, et al. Efficacy of different surgical approaches in the clinical and survival outcomes of patients with early-stage cervical cancer: protocol of a phase III multicentre randomised controlled trial in China. BMJ Open 2019;9:e029055.

37 Liu Y, Li L, Wu M, et al. The impact of the surgical routes and learning curve of radical hysterectomy on the survival outcomes in stage Ib cervical cancer: a retrospective cohort study. Int J Surg 2019;68:72-7.

38 Conrad LB, Ramirez PT, Burke W, et al. Role of minimally invasive surgery in gynecologic oncology: an updated survey of members of the Society of gynecologic oncology. Int J Gynecol Cancer 2015;25:1121-7.

39 Nezhat F. Minimally invasive surgery in gynecologic oncology: laparoscopy versus robotics. Gynecol Oncol 2008;111:S29-32.

40 Seamon LG, Fowler JM, Richardson DL, et al. A detailed analysis of the learning curve: robotic hysterectomy and pelvic-aortic lymphadenectomy for endometrial cancer. Gynecol Oncol 2009;114:162-7.

41 Estape R, Lambrou N, Diaz R, et al. A case matched analysis of robotic radical hysterectomy with lymphadenectomy compared with laparoscopy and laparotomy. Gynecol Oncol 2009;113:357-61.

42 Chong GO, Park NY, Hong DG, et al. Learning curve of laparoscopic radical hysterectomy with pelvic and/or para-aortic lymphadenectomy in the early and locally advanced cervical cancer: comparison of the first 50 and second 50 cases. Int J Gynecol Cancer 2009:19:1459-64.

43 Reade C, Hauspy J, Schmuck M-L, et al. Characterizing the learning curve for laparoscopic radical hysterectomy: buddy operating as a technique for accelerating skill acquisition. Int J Gynecol Cancer 2011;21:930-5.

44 Kong T-W, Chang S-J, Paek J, et al. Learning curve analysis of laparoscopic radical hysterectomy for gynecologic oncologists without open counterpart experience. Obstet Gynecol Sci 2015:58:377-84.

45 Hwang JH, Yoo HJ, Joo J, et al. Learning curve analysis of laparoscopic radical hysterectomy and lymph node dissection in early cervical cancer. Eur J Obstet Gynecol Reprod Biol 2012;163:219-23.

46 Pergialiotis V, Rodolakis A, Christakis D, et al. Laparoscopically assisted vaginal radical hysterectomy: systematic review of the literature. J Minim Invasive Gynecol 2013;20:745-53.

$47 \mathrm{Kim} \mathrm{SI}$, Cho JH, Seol A, et al. Comparison of survival outcomes between minimally invasive surgery and conventional open surgery for radical hysterectomy as primary treatment in patients with stage IB1-IIA2 cervical cancer. Gynecol Oncol 2019;153:3-12.

48 Kohler C, Hertel H, Herrmann J, et al. Laparoscopic radical hysterectomy with transvaginal closure of vaginal cuff - a multicenter analysis. Int J Gynecol Cancer 2019;29:845-50.

49 Pecorelli S, Zigliani L, Odicino F. Revised FIGO staging for carcinoma of the cervix. Int J Gynaecol Obstet 2009;105:107-8.

50 Ruckdeschel JC, Finkelstein DM, Ettinger DS, et al. A randomized trial of the four most active regimens for metastatic non-small-cell lung cancer. J Clin Oncol 1986;4:14-22.

51 Piver MS, Rutledge F, Smith JP. Five classes of extended hysterectomy for women with cervical cancer. Obstet Gynecol 1974;44:265-72.

52 Querleu D, Morrow CP. Classification of radical hysterectomy. Lancet Oncol 2008:9:297-303.

53 Pedone Anchora L, Turco LC, Bizzarri N, et al. How to select early-stage cervical cancer patients still suitable for laparoscopic radical hysterectomy: a Propensity-Matched study. Ann Surg Oncol 2020;27:1947-55.

54 Obermair A, Gebski V, Frumovitz M, et al. A phase III randomized clinical trial comparing laparoscopic or robotic radical hysterectomy with abdominal radical hysterectomy in patients with early stage cervical cancer. J Minim Invasive Gynecol 2008;15:584-8.

55 National Cancer Institute. Common terminology criteria for adverse events (CTCAE) v4.03. Available: https://ctep.cancer.gov/ protocolDevelopment/electronic_applications/ctc.htm\#ctc_50 